Société d'histoire de la révolution de 1848 et des

révolutions du XIXe siècle

\title{
Le « Robert et Cougny » et l'invention des parlementaires
}

Biographical dictionaries and the social identity of the French deputies

\section{Herve Fayat et Nathalie Bayon}

\section{OpenEdition}

\section{Journals}

Édition électronique

URL : http://journals.openedition.org/rh19/1144

DOl : $10.4000 /$ rh 19.1144

ISSN : $1777-5329$

\section{Éditeur}

La Société de 1848

\section{Édition imprimée}

Date de publication : 1 décembre 2006

Pagination : 55-78

ISSN : 1265-1354

Référence électronique

Herve Fayat et Nathalie Bayon, «Le "Robert et Cougny » et l'invention des parlementaires », Revue d'histoire du XIXe siècle [En ligne], 33 | 2006, mis en ligne le 01 décembre 2008, consulté le 21 avril 2019. URL : http://journals.openedition.org/rh19/1144 ; DOI : 10.4000/rh19.1144 


\title{
HERVE FAYAT ET NATHALIE BAYON
}

\author{
Le "Robert et Cougny" \\ et l'invention des parlementaires ${ }^{1}$
}

Lorsque l'histoire rencontre des groupes explicites, institués dans leur dénomination, leurs frontières et leurs fonctions, l'évidence de leur existence juridique et sociale est parfois si forte qu'elle s'impose à l'analyste sommé de découvrir la substance sociale du groupe plutôt que de mettre au jour le travail d'institutionnalisation et d'unification auquel ses membres doivent la stabilité et la cohésion de leur identité collective. C'est pourquoi les populations construites par - et pour - les grandes institutions (armée, Église, grands corps d'État ${ }^{2}$ ou assemblées représentatives) soumettent, avec une intensité particulière, l'analyste à un dilemme récurrent en sciences sociales; tandis qu'il lui faut se détacher d'une définition préalable qui naturalise la position qu'elle décrit et fige le système d'interrogation auquel elle est soumise, il doit, simultanément, réintroduire la constitution de ce point de vue institué dans la sociogenèse de la position qui s'y rapporte et dans l'explicitation des investissements sociaux qu' elle suscite.

Les parlementaires, population instituée pour laquelle le «nominal du groupe» joue un rôle essentiel dans l'existence sociale de ses membres ${ }^{3}$, fournissent un exemple de cette difficulté posée au politiste et à l'historien par la

1. Cette recherche trouve son origine dans une enquête prosopographique menée par le Groupe

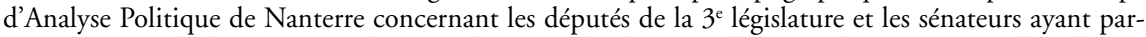
ticipé au vote des lois communales. Conduite sous la direction de Bernard Lacroix et Willy Pelletier, cette recherche a rassemblé Jean-Claude Bussière, Éric Guichard, Marie-Hélène Lechien, Christophe Le Digol, Laurent Quéro, Guillaume Sacriste, Christophe Voilliot, Laurent Willemez et nous-mêmes. Le travail de collecte des données était tributaire, dans un premier temps tout au moins, des informations recueillies dans les dictionnaires biographiques. Or, le rapport sous lequel nous envisagions cette population de parlementaires ne trouvait pas d'équivalent dans ces sources dont le contenu était erroné (comme nous avons pu le constater en collaborant à l'enquête de l'URA 1016) ou dissonant, chacun d'entre eux semblant orienté par une entreprise d'objectivation sociale particulière. Dès lors, nous avons affectés à deux objets complémentaires ces dictionnaires : la sociogenèse puis la sociographie de la population étudiée.

2. Pierre Bourdieu, La noblesse d'État, Paris, Minuit, 1989, 568 p.

3. Il va de soi que l'enjeu de nomination est commun à tous les groupes dont l'existence sociale, comme le montrait Luc Boltanski pour les cadres, ne peut reposer sur une définition technique. Pour des groupes non institués comme les intellectuels, la nomination collective engage tant l'existence sociale des membres que le travail de définition et de délimitation du groupe est lui-même inclus dans l'activité de ceux qui agissent sous ce label. Le Dictionnaire des intellectuels français, Jacques Julliard, Michel Winock [dir.], Paris, Seuil, 1996, 1258 p., atteste de l'actualité de ce travail symbolique. 
prégnance d'une définition préalable. Du reste, en dépit des variations pratiques de l'activité parlementaire qu'elle reconnait ${ }^{4}$, l'historiographie parlementaire s'est jusqu'à présent inclinée devant l'identité nominale d'un groupe auquel elle prête une unité historique. Cette forme de réification, courante à l'égard de personnes instituées ${ }^{5}$, procède de la reconduction d'un point de vue établi par lequel l'analyste attribue à tous les députés des intérêts et des réalisations communes et rabat sur ces populations un système d'interrogation invariant; ce qui revient à considérer les députés de toutes les époques comme les ressortissants d'une "institution réalisée». Bien sûr, chacun sait qu'un député au Corps législatif n'est pas un député de la Troisième République, qu'un notable differe d'un professionnel, que Thivrier n'est pas Deschanel, mais voilà, les députés " existent", et l'on devine mal quel négationnisme sociologique pourrait effacer leur existence légale ou négliger cette croyance sociale. C'est pourquoi, loin d'escamoter cette unité du groupe - produit et enjeu implicite du processus d'autonomisation d'une sphère de la représentation politique - nous traiterons, au travers de la consultation sociologique des annuaires de parlementaires, de ce travail d'unification qui est constitutif de la réalité sociale de la position parlementaire.

Les dictionnaires de "parlementaires» illustrent en effet la variété d'enjeux sociaux et savants attachés aux usages d'un substantif marqué tout à la fois par une invariance que contredit la diversité historique des personnages sociaux et des formes d'activités qu'il héberge, et par une ambivalence qui fait simultanément de ce vocable un outil de désignation d'une population et un instrument de délégation en sa faveur.

Tout comme les manuels et recueils d'éloquence parlementaire, les essais politiques, les portraits ou chroniques parlementaires, ces dictionnaires traduisent l'existence de ce que l'on peut nommer des «luttes d'autonomisation " ${ }^{6}$ pour qualifier les «luttes biographiques ${ }^{7}$ bien sûr, mais aussi toutes les luttes de définition des conditions et des formes de la représentation poli-

4. De nombreux travaux sont aujourd'hui disponibles concernant l'analyse des relations de représentation et d'autres sont en cours sur la division du travail parlementaire, les moyens matériels et bureaucratiques grâce auxquels il est réalisé, les répertoires d'action spécifiques dans lesquels il se concrétise à chaque époque, les transactions ou regroupements auxquels il donne lieu, les mœurs politiques auxquels il prédispose, l'intérêt public qu'il suscite... Tous concluent à une diversité des conditions historiques d'exercice de l'activité parlementaire.

5. Sur l'analyse sociologique de personnes instituées, voir Bernard Lacroix et Jacques Lagroye [dir.], Le président de la République, Paris, Fondation nationale des sciences politiques, 1992, 402 p.

6. Ces luttes symboliques traduisent un état intermédiaire du jeu politique du point de vue de sa spécialisation, de la structure de sa concurrence et des intérêts sociaux qu'il suscite. Impliquant souvent des tiers acteurs et portant sur les formes et les conditions de la représentation, elles supposent et construisent l'autonomie de l'espace dont elles classent les personnes et les produits. Le meilleur exemple nous est fourni par les recueils d'éloquence qui, attestant de l'autonomisation d'une éloquence parlementaire, produisent les schèmes d'appréciation de ce genre nouveau qu'ils distinguent de l'éloquence du barreau ou de la chaire, voir, Hervé Fayat, "Discourir ", dans Vincent Duclert, Christophe Prochasson [dir.], Dictionnaire critique de la République, Flammarion, 2002, 1340 p.

7. Sur cette notion, voir Michel Offerlé, "Introduction", La profession politique XIXe-XXe siècle, Paris, Belin, 1999. 363 p 
tique dont les députés sont à la fois les sujets et les objets, particulièrement dans les premières décennies de la Troisième République. En effet, tout en fournissant des informations biographiques sur les représentants à l'attention des citoyens, des publicistes, des hommes d'affaires, etc., ces annuaires biographiques concurrents unifient et organisent symboliquement la population qu'ils répertorient; en d'autres termes, chacun d'entre eux propose une "gestion symbolique» des parlementaires. Sans exagérer le rôle tenu par ces répertoires dans l'unification des parlementaires et dans la fixation de leurs attributs légitimes, il est permis de penser que ces entreprises de définition de la position parlementaire ne sont ni totalement arbitraires ni entièrement inopérantes, tout au moins quant à l'imposition du point de vue sous lequel les parlementaires doivent être envisagés et quant à la légitimation de leur propriétés sociales ${ }^{8}$. C'est pourquoi, consultés dans la logique de construction de la population qu'ils décrivent, ces répertoires peuvent contribuer à une analyse sociogénétique des parlementaires aussi efficacement, sinon plus, qu'ils ont concouru à leur sociographie.

L'intérêt de cette enquête sur la production des annuaires de parlementaires réside d'abord dans le changement de logique de consultation à laquelle elle convie les utilisateurs de ces documents. Prendre les dictionnaires de parlementaires pour objet, y chercher non plus des données biographiques mais des principes de classement des propriétés sociales des députés conduit à ne plus y voir des sources mais des produits et/ou des instruments du processus d'autonomisation de l'activité parlementaire et d'affirmer, par là même, une série de ruptures utiles à l'historiographie parlementaire, à commencer par l'objectivation du point de vue véhiculé par ces «sources».

Le second mérite tient à la rupture que cette lecture sociologique des dictionnaires de parlementaires permet d'introduire à l'égard de la définition instituée des parlementaires. Cette posture invite en effet à inverser la logique de recherche qui faisait prévaloir la sociographie des parlementaires sur leur sociogenèse et, en outre, elle engage cette dernière sur le terrain encore peu exploré des principes d'unification de cette population. En portant au jour les tâtonnements et les divergences quant aux principes de définition de l'activité parlementaire, cette historicisation invite à penser, à l'encontre des visions objectivistes et naturalistes, que l'identité sociale de ces derniers demeurait encore floue à l'orée du vingtième siècle. Il va de soi que cette observation est transposable à d'autres populations instituées dont les moyens d'affirmation et les principes de définition ne sont jamais aussi solennels, lointains et unanimes que l'anamnèse officielle ne le laisse entendre.

8. Même si l'objectif n'est pas explicite, le travail de légitimation est bien présent dans ces recueils et suggère des rapprochements avec l'activité de valorisation politique des attributs ouvriers constatée par Michel Offerlé "Illégitimité et légitimation du personnel politique ouvrier en France avant 1914 ", dans Annales ESC, volume 39, n 4, 1984, p. 681-713, ou par Bernard Pudal, Prendre Parti, Paris, Fondation nationale des sciences politiques, 1989, $329 \mathrm{p}$. 
Dans une période marquée par le recours à la prosopographie, il apparaît ensuite qu'une réflexion sur les principes constitutifs d'une population permet de combattre la prégnance des définitions préalables, jamais aussi forte qu'à l'occasion des enquêtes sur les origines au cours lesquelles la régression de la personne instituée vers la personne concrète prédispose tout à la fois à rechercher les traits constitués et à naturaliser les propriétés d'origine. Autrement dit, à s'enfermer dans un système d'interrogation tautologique cherchant ses réponses dans les sources auxquelles il doit sa possibilité. Cette enquête interpelle également la méthode prosopographique sur sa propension à réduire un groupe à l'agrégation des propriétés moyennes ou modales de ses membres alors que l'analyse d'une entreprise de "gestion symbolique» des parlementaires tente de saisir un aspect de son processus de regroupement ${ }^{9}$.

Ensuite, l'unification symbolique réalisée par ces annuaires - particulièrement par le «Robert et Cougny» - est loin d'être un artifice car elle soutient la valeur faciale de la position parlementaire grâce à laquelle son titulaire peut se prévaloir d'une communauté de statut avec ses pairs comme avec ses aînés, quelles que soient ses propriétés biographiques et les circonstances dans lesquelles elles sont en jeu. En établissant cette unité historique de la fonction parlementaire, ces ouvrages - sortes de contreparties symboliques de la diversification sociale du recrutement parlementaire et de la multi-positionnalité de plus en plus appelée et promise par les activités de représentation - produisent une facette essentielle la position parlementaire.

Enfin, ces annuaires, véritables instruments de légitimation et de délégitimation de propriétés sociales, mettent en scène les principes sociaux de la délégation politique dont ils éclairent les enjeux.

Le choix du "Robert et Cougny" pour illustrer ce travail d'objectivation sociale tient à sa notoriété, à sa postérité, à son époque de publication (1889) et surtout à son projet de totalisation et d'unification de l'activité parlementaire qui le distingue des autres annuaires parlementaires. Afin de rendre compte de l'étendue des opérations réalisées par cet ouvrage et d'illustrer certaines de ses propriétés, nous exposerons successivement sa position dans l'espace de représentation des parlementaires, les dispositions de ses auteurs et les circonstances de leur travail, les figures parlementaires qu'il constitue avant d'évoquer les usages sociaux et savants qui font de ce dictionnaire une "objectivation réussie».

9. Pour une réflexion sur la prosopographie, voir Bernard Lacroix, «Six observations sur l'intérêt de la démarche prosopographique dans le travail historiographique», dans Jean-Marie Mayeur, Jean-Pierre Chaline et Alain Corbin [dir.], Les parlementaires de la Troisième République, Paris, Publications de la Sorbonne, 2003, p. 27-42. 
LE «Robert \& COUgny » DANS LE «Siècle Des DiCtionnaires »

Plus personne ne conteste l'autorité de l'institution parlementaire en matière de «gestion biographique» des représentants. Cette attribution est d'ailleurs concédée à de nombreuses organisations qui entretiennent leur «façade institutionnelle» par le façonnage des biographies de leurs membres au travers de trombinoscopes, d'annuaires des personnels, bref, de toutes sortes de documents publics par lesquels les modalités de présentation de soi sont accordées aux positions instituées ${ }^{10}$. Au XIX ${ }^{e}$ siècle, les assemblées représentatives n'exerçaient pas ce «monopole naturel» de production des images publiques des députés et des sénateurs. Le "siècle des dictionnaires" ${ }^{11}$ était aussi celui des biographies collectives et l'édition des annuaires parlementaires s'effectuait hors du contrôle des Chambres. Malgré leur place secondaire sur le marché des biographies collectives, ces publications attestent de la vigueur des «luttes d'autonomisation» et de la diversité des principes de définition appliqués à la population parlementaire jusqu'à l'orée du vingtième siècle. Le "Robert et Cougny» marque justement un point de retournement au-delà duquel cette production s'inscrivit dans une tout autre configuration éditoriale caractérisée par une raréfaction des éditeurs et des parutions et par une plus grande emprise de la Chambre. Cette monopolisation de la "gestion biographique» s'accompagna d'une pacification des luttes de définition à laquelle le travail d'unification symbolique effectué par le "Robert et Cougny" n'est certainement pas étranger. En résumé, le Dictionnaire des parlementaires traduit une autonomisation du champ de représentation des parlementaires que l'on peut observer en le rapportant successivement aux grandes biographies collectives puis aux annuaires homologues.

Les dictionnaires ou annuaires de parlementaires partagent une communauté de genre avec les nombreuses biographies collectives du XIX siècle; nobiliaires, annuaires ecclésiastiques, mondains ou professionnels abondaient ${ }^{12}$, leur production mobilisait des moyens considérables ${ }^{13}$ et ils rencontraient un public nombreux ${ }^{14}$. Sans entrer dans les multiples raisons de la

10. Comme exemple d'analyse des biographies d'institution, on peut se reporter à Éric Agrikolianski, "Biographies d'institution et mise en scène de l'intellectuel. Les candidats au comité central de la Ligue des droits de l'homme entre 1945 et 1975", dans Politix, 1992, n²7, p. 94-110.

11. Le Siècle des dictionnaires : exposition, Paris, Musée d'Orsay, 25-30 août 1987, Paris, Réunion des musées nationaux, 1987.

12. La consultation de la Bibliographie de la France permet de se faire une idée de l'abondance de cette production dont le dénombrement est difficile car on ne sait quelles limites assigner à ce genre et, de plus, beaucoup de ces publications n'émargent pas sous le terme d'annuaire, de biographie ou de dictionnaire de sorte que leur repérage exhaustif est un défi.

13. On demeure frappé par le nombre de collaborateurs mobilisés dans ces entreprises; pas moins d'une quarantaine pour la Biographie bretonne de Levot tandis que Le Dictionnaire de la Révolution et de l'Empire dirigé par Robinet a nécessité quant à lui une vingtaine de collaborateurs.

14. Élisabeth Parinet, Une histoire de l'édition à l'époque contemporaine : XIXé-XXe siècle, Paris, Seuil, 2004,489 p. 
prospérité d'un genre qui signale certainement un intense travail de regroupement ${ }^{15}$, les biographies collectives occupèrent longtemps une place de choix dans l'univers de l'édition, tant par la quantité et la variété des ouvrages publiés ${ }^{16}$ que par la rétribution de leurs auteurs. Ainsi, Gustave Vapereau perçut, dès la première édition de son Dictionnaire universel des contemporains, trois fois plus de droits que n'en obtint Michelet pour son Histoire de la Révolution française ${ }^{17}$, soit un montant presque équivalent à ce que reçut Eugène Sue pour la publication en feuilleton des Mystères de Paris... Ce rang dans la hiérarchie pécuniaire des genres se doublait d'une place éditoriale considérable puisque, vers la fin du Second Empire, les dictionnaires biographiques représentaient à eux seuls un quart des publications historiques ${ }^{18}$.

Malgré son succès public et l'exhibition des titres de certains de ses rédacteurs ${ }^{19}$, ce genre était souvent contesté voire méprisé par les historiens et, si les préfaces affichaient souvent une ambition historique s'ajoutant à la prétention d'exhaustivité des fiches biographiques, c'était surtout pour se dédouaner du soupçon d'approximation et de plagiat qui entachait ces ouvrages ${ }^{20}$. Ainsi, les tentatives pour élever la biographie au rang de la «science historique», pour en faire une "sœur cadette» ou une branche complémentaire de l'histoire qui aurait pour "objet l'individu tout entier" alors que "l'histoire garderait les masses pour domaine ${ }^{21}$ paraissent aujourd'hui vaines.

Cependant, bien en deçà de ces proclamations, se constitue bel et bien une pratique de la biographie collective avec ses principes narratifs et ses topiques sociaux qui, prosaïquement, circulent d'un ouvrage à l'autre avec les informations biographiques qu'ils organisent, jusqu'à former une sorte d'implicite du genre reliant entre elles les entreprises les plus diverses. C'est pourquoi ces répertoires de dominants et de principes de domination pouvaient fournir des modèles de représentation et de légitimation disponibles

15. Sous couvert de fournir des informations utiles au groupe duquel elles tirent leur justification, ces entreprises s'inscrivent toutes dans une concurrence particulière pour la définition de la "bonne forme" d'un groupe. C'est pourquoi leur apparition, leur nombre, leur forme, leur contenu, la qualité de leurs rédacteurs, etc. sont autant d'indicateurs de rassemblement du groupe auquel elles s'adressent.

16. Jules Auffray, Bibliographie des recueils biographiques de contemporains aux $X I X^{e}$ et $X X^{e}$ siècles en France, mémoire pour le diplôme de l'Institut National des Techniques Documentaires, 1963.

17. Christophe Charle, "Le champ de la production littéraire», dans Roger Chartier et Henri-Jean Martin [dir.], Histoire de l'édition française, Paris, Fayard, Cercle de la librairie, 1989, p. 162.

18. Théodore Zeldin, "Biographie et psychologie sous le Second Empire», dans Revue d'histoire moderne et contemporaine, tome XXI, janvier-mars 1974, p. 58-74. Dans cette estimation Théodore Zeldin écarte les dictionnaires régionaux, spécialisés et professionnels, ce qui amoindrit considérablement leur part relative.

19. Les plus grandes biographies, comme celle de Louis-Gabriel Michaud et de Gustave Vapereau, affichent un aréopage de savants et d'érudits dont beaucoup "préferent rester dans l'anonymat" au dire de Vapereau. Mais il est cependant exact qu'au milieu du siècle encore, ces ouvrages réunissaient d'éminents collaborateurs rédigeant quelques fiches dans leur domaine de compétence (Arago, par exemple).

20. Comme pour réfuter par avance les accusations de plagiat, toutes les biographies, y compris le "Robert et Cougny", arborent les principes documentaires auxquels elles se sont conformées et relèvent fièrement les erreurs décelées chez leurs devancières.

21. Termes employés par Charles Nodier dans son discours préliminaire à la seconde édition de la Biographie universelle ancienne et moderne, Paris, A. Thoisnier Desplaces éditeur, 1848, p. 9. 
pour caractériser les parlementaires, dans une époque d'incertitude quant aux catégories d'appréhension d'une activité encore peu spécialisée jusqu'au milieu du dix-neuvième siècle ${ }^{22}$. À l'époque de la publication du "Robert \& Cougny", les grandes biographies semblaient en déclin, mais cet ouvrage demeure lié à ce travail protéiforme auquel il emprunte et auquel il contribue abondamment ${ }^{23}$ tout en affirmant l'autonomie d'un type d'annuaire auquel il convient maintenant de le rapporter.

Les annuaires parlementaires, dont on trouve des exemplaires dès la Révolution, connaissaient une diffusion plus restreinte que les grandes entreprises biographiques qui viennent d'être évoquées et leur publication appelle à son tour plusieurs remarques.

On constate tout d'abord une sorte de nationalisation de ces publications qui, pour une bonne part d'entre elles, demeuraient régionales jusqu'à la Troisième République. On observe ensuite que leur fréquence de publication coïncide assez clairement avec les événements politiques majeurs du $\mathrm{XIX}^{\mathrm{e}}$ siècle : la Seconde République de même que les débuts de la Troisième République ${ }^{24}$ connurent une floraison d'ouvrages de ce type et la cinquième législature, celle du "Robert et Cougny", vit la sortie de onze annuaires parlementaires! Un paroxysme suivi d'une lente mais inexorable décrue. Ce rythme accrédite une co-occurrence entre la publication de ces dictionnaires et les circonstances dans lesquelles la légitimité d'un nouveau personnel parlementaire était en cause ou lorsqu'un soupçon d'illégitimité frappait l'ensemble de la représentation, comme à l'époque du boulangisme. La raréfaction des dictionnaires de parlementaires à compter du tournant du siècle correspondrait, quant à elle, à une sorte d'apaisement des luttes de définition de la fonction parlementaire.

Enfin, si les auteurs et les éditeurs d'annuaires parlementaires n'espéraient pas une diffusion comparable à celle des grandes biographies, ils pouvaient tout au moins compter sur l'intérêt pratique d'un public impliqué, à un titre ou à un autre, dans une société parlementaire en expansion. De plus, la rela-

22. Ces ouvrages accumulent, tous ensemble, un stock d'informations biographiques et de principes d'identification sociale des classes dominantes offerts à une circulation par laquelle, pris isolément, ils conservent une certaine autonomie vis-à-vis des groupes dont ils se recommandent. Cette autonomie est à l'origine d'un décalage entre les topiques sociaux utilisés par le «Robert et Cougny» et les classements autochtones; c'est d'ailleurs par cet écart que l'on peut mettre en évidence les propriétés relationnelles de tout travail d'objectivation sociale.

23. On remarquera que bien des fiches sont "inspirées» du Dictionnaire universel de la noblesse de même que l'on est troublé par la similitude entre certaines fiches du «Robert et Cougny» et du Dictionnaire national des contemporains de Curinier, paru dix ans plus tard...

24. On compte 20 annuaires parlementaires parus en 1871 puis, à compter de la seconde législature (1877-1881), leur fréquence devint plus faible, 5 pour la seconde, 2 pour la troisième, 4 pour la quatrième, 11 pour la cinquième (le Robert et Cougny paraît durant cette législature 1889 - 1893), 7 pour la sixième et 6 dans la législature du tournant du siècle. Ensuite, la publication d'annuaires parlementaires s'établira autour de 2 ou 3 seulement par législature jusqu'aux années 1920 . Nous avons établi ces comptes à partir de Huguette-Perinne Marcheix, Bibliographie analytique de biographies des parlementaires français de 1871 à 1960, mémoire pour le diplôme de l'Institut National des Techniques Documentaires, 1965. 
tive intégration sociale du public concerné, la modeste pagination, la facilité de réalisation et la rapidité de vente de ces ouvrages de début de législature plaçaient ces entreprises à la portée de petits éditeurs. Du reste, la recension fait apparaître une prédominance sur ce marché des petites maisons d'édition escomptant certainement un bref retour sur investissement ${ }^{25}$.

Tel est le contexte éditorial de ce type d'ouvrages, mais l'occasion du centenaire de la Révolution et la perspective d'être épaulé par la Chambre autorisaient une entreprise plus ambitieuse, comme le Dictionnaire des parlementaires français. Cependant, le projet exigea un travail de collecte de données biographiques important, pour lequel Bourloton engagea deux copistes réguliers; peut-être est-ce pour amortir ces dépenses qu’il publia en même temps un autre annuaire parlementaire préfacé par Deschanel ${ }^{26}$ puis, en 1898, une biographie collective des parlementaires vendéens ${ }^{27}$. Bien que publié par un petit éditeur, le "Robert et Cougny» ne marque pas moins une rupture dans le genre duquel il relève. Par son volume (cinq tomes), par son amplitude historique (un siècle) et enfin par sa proximité avec l'institution parlementaire, il réalisa un travail d'unification sans précédent et anticipa quelque peu sur les biographies d'institution qui sont le destin de ce genre. Par tous ces aspects, il occupe une position intermédiaire dans le processus d'autonomisation de l'écriture et de l'édition des biographies de députés.

\section{LES AUTEURS}

L'écriture du «Robert \& Cougny» est soutenue par des dispositions acquises par les auteurs au cours d'une trajectoire sociale qui les rapprocha de l'institution parlementaire et les prédisposa à voir dans l'histoire de cette institution la réalisation de l'idée républicaine qui, en cette fin des années 1880, leur semblait mériter d'être défendue ou, tout au moins, commémorée dans le cadre du centenaire de la Révolution ${ }^{28}$

Loin d'être singulier, le parcours de ces auteurs est représentatif du flux dans lequel furent entraînés certains clercs en mesure de négocier leurs compétences auprès d'institutions publiques (enseignement, municipalités, administration centrale, Chambre des députés, etc.) dont le développement transformait la structure des positions qui leur étaient offertes après l'Empire. Parmi les auteurs de biographies parlementaires certains profitèrent de la multiplication de ces positions pérennes ou temporaires adossées à la

25. Mis à part certains éditeurs importants, comme Dentu ou Garnier, les petits éditeurs souvent provinciaux dominent ce marché.

26. Edouard Duguet, Les députés et les cahiers électoraux de 1889, Paris, Bourloton, 1890, 423 p.

27. Edgar Bourloton, Cent ans de législature. Les députés de la Vendée depuis 1789, Fontenay-le-Comte, Henry Cormeau, 1898, 129 p.

28. Rappelons que la sortie du Dictionnaire des parlementaires français en 1889 est liée à l'organisation du centenaire. 
construction de l'État parlementaire : tel est le cas de Jules Clère longtemps employé par la Chambre ou de Louis Gustave Vapereau successivement secrétaire de Victor Cousin, enseignant puis préfet du Cantal ${ }^{29}$

Polygraphe et éditeur, Edgar Bourloton est né à Fontenay-le-Comte en 1844, dans cette portion républicaine de la Vendée. Son père, professeur de rhétorique au collège de Fontenay, écrivait des ouvrages scolaires ${ }^{30} \mathrm{et}$, malgré les attaches catholiques de la famille ${ }^{31}$, Edgar Bourloton manifesta des opinions plutôt républicaines qui l'opposèrent assez vite à son entourage ${ }^{32}$. À la suite de la défaite de Sedan, il écrivit un récit de voyage sur l'Allemagne ${ }^{33}$ où il fut prisonnier puis, en collaboration avec Edmond Robert ${ }^{34}$ (préfet de Vendée), un essai politique sur la Commune ${ }^{35}$. Le texte sur l'Allemagne est en réalité une sorte de manifeste politique s'inscrivant dans le sillage des interrogations douloureuses qui suivirent la défaite de 1870 . On y découvre un engagement politique sans ambiguïté comme le montrent les extraits suivants : comparant la France et l'Allemagne, il écrit «la France purifiée si cruellement de vingt années d'absolutisme n'a à redouter aucune comparaison"; sur le choix de régime, il ajoute "ce n'est pas au triomphe de telle ou telle forme de gouvernement que les efforts doivent tendre mais au progrès des institutions sociales»; sur les libertés formelles, et il déclare "nos voisins nous empruntaient non pas les stériles arguments de Voltaire [...], mais les simples et fécondes idées de Rousseau, le vrai révolutionnaire et seul ami du peuple». Plus loin il écrit «la liberté d'écrire, de dire, de se réunir n'est qu'un trompe l'œil tant que la législation entrave la liberté de faire...", et enfin la souveraineté n'est à ses yeux "qu'un mot tant qu'elle se concentre entre les mains de la classe riche».

Outre ces textes de jeunesse, il écrivit jusqu’à sa mort en 1914 des chroniques historiques sur Fontenay et sa région durant les guerres de Vendée ainsi que de nombreuses biographies d'ecclésiastiques dans la Revue du Bas-Poitou ${ }^{36}$ dirigée par René Valette. Ce savoir-faire biographique fut bien sûr recy-

29. L'homologie sociale entre ces deux personnages et nos trois auteurs est troublante. Ainsi, Jules Clère fut publiciste (La Réforme, Le Courrier français puis le National) avant d'entrer à l'Assemblée Nationale en tant que sécrétaire-rédacteur de commission parlementaire (particulièrement auprès de la commission des douanes d'après son dossier de pension) et avant la publication de sa biographie des députés puis des sénateurs, il écrivit lui aussi un Annuaire des hommes de la Commune en 1871.

30. La correspondance du père d'Edgar Bourloton montre des relations suivies avec des libraireséditeurs parisiens spécialisés dans l'édition d'ouvrages scolaires destinés à l'enseignement élémentaire comme Debrozy et Magdeleine (Archives départementales de la Vendée, fonds Gambier, cote 52 J 40).

31. Son frère devint zouave pontifical et Edgar Bourloton lui-même garda toujours un intérêt très marqué pour le fait religieux.

32. Une série d'échanges épistolaires, alors qu'il effectuait ses études de droit à Paris dans les années 1860, montre le désaccord politique qui l'opposait à l'abbé Pondevie, son ancien aumônier (Archives départementales de la Vendée, fonds Gambier, cote 52 J 186).

33. Edgar Bourloton, L'Allemagne contemporaine, par Edgar Bourloton, engagé volontaire de 1870 aux zouaves de la Garde, Paris, Baillière, 1873, viii-284 p.

34. Signalons qu'Edmond Robert est également l'auteur d'un dictionnaire des préfets... 1873 .

35. Edgar Bourloton et Edmond Robert, La Commune et ses idées à travers l'histoire, Paris, Baillière,

36. La Revue du Bas-Poitou est une revue bimestrielle qui paraît sous ce titre de 1888 à 1961 avant de 
clé dans l'entreprise du Dictionnaire des parlementaires. Bourloton était par ailleurs gérant du Bulletin Hebdomadaire de Statistique Municipale de la ville de Paris dirigé par Jacques Bertillon ${ }^{37}$. On peut envisager que cette fréquentation de la statistique sanitaire et sociale publique ait favorisé une disposition à construire des populations, à leur imputer certaines propriétés et à découvrir une vertu scientifique à cette opération classificatoire. Un article d'Edgar Bourloton sur l'espérance de vie des parlementaires ${ }^{38}$ démontre d'ailleurs la croyance de l'auteur dans l'unité sociale et même "physiologique» de ce groupe. Indexer des propriétés physiologiques sur une appartenance sociale n'a rien d'aberrant en soi - voir l'usage actuel des catégories socio-professionnelles - mais cela suppose une existence sociale du groupe bien établie... à moins que ce ne soit, là encore, un moyen de l'attester sur le modèle que Jacques Bertillon élaborait au même moment ${ }^{39}$.

Gaston Cougny, quant à lui, était professeur d'histoire de l'art auprès des écoles municipales de Paris et écrivit de nombreux ouvrages destinés à l'enseignement artistique. Il fut également avocat à la cour d'appel de Bourges et se présentait régulièrement comme publiciste. Or, le corps enseignant de la ville de Paris (alors dirigée par la gauche) était partie prenante des enjeux historiographiques et politiques opposant radicaux et opportunistes pour la définition d'une doctrine républicaine. Par ailleurs, Gaston Cougny, dont le père fut conseiller général, entretenait lui-même une activité politique assidue auprès des radicaux-socialistes du Cher dès les années 1880 . Il se présenta d'ailleurs à la députation lors de l'élection législative de 1885 sur une liste où figuraient Maret, Pyat et Vaillant... Plusieurs fois candidat malheureux à la députation dans la première circonscription du Cher par la suite, les archives départementales montrent qu'il livra des campagnes pugnaces durant lesquelles il bénéficia du soutien actif de personnalités nationales comme Coutant, Groussier ou Viviani ${ }^{40}$. Tandis qu'il évoluait vers le socialisme, il fut élu conseiller municipal de Bourges en 1900 où il fut chargé de

devenir, de 1962 à 1972, la Revue du Bas-Poitou et des provinces de l'ouest. Nous avons pu y recenser plus d'une trentaine de chroniques publiées sous son nom.

37. En réalité, Bourloton était gérant et éditeur de cette publication dont la distribution est effectuée par la librairie Masson. Cette statistique sanitaire a joué un grand rôle dans la construction d'une statistique d'État et, comme on sait, dans la documentation du livre de Louis Chevalier, Classes laborieuses, classes dangereuses, Paris, Plon, 1958, 566 p.

38. Edgar Bourloton, La longévité des parlementaires, Paris, La vie contemporaine, 1911, 11 p. Dans ce texte, où il démontre une rare fascination pour les statistiques démographiques, il se livre à de nombreux calculs par lesquels il établit que les parlementaires ont une espérance de vie de loin supérieure à la moyenne française, et même aux «professions intellectuelles», affirme-t-il.

39. Au même moment, Jacques Bertillon présente au congrès de Chicago deux communications dans lesquelles il expose ses principes de classification professionnelle en lien avec les pathologies. Voir Alain Desrosières, La politique des grands nombres, Paris, La Découverte, 1993, 437 p.

40. Les rapports de police nous apprennent qu'il développe régulièrement les thèmes du programme socialiste lors de ses réunions publiques (impôts sur le revenu, retraites ouvrières, etc. pour lesquelles il signe à l'occasion un mandat impératif) au cours desquelles il organise des collectes pour les verriers d'Albi (Archives Départementales du Cher, série $20 \mathrm{M}, \mathrm{n}^{\circ} 38$ ). 
l'enseignement jusqu'en 1902, date de révocation du conseil municipal ${ }^{41} \ldots$ et de son retrait de la vie politique à la suite d'un nouvel échec aux législatives de 1902 en tant que candidat du parti socialiste français (PSF). Les propos tenus par le maire de Vierzon pour son éloge funèbre - «un artiste égaré en politique» - illustrent les relations passionnées et douloureuses que Gaston Cougny entretenait à l'égard de l'univers politique dans lequel il n'était pas si obscur puisque le journal L'Humanité lui consacra une nécrologie lors de son décès en 1906.

Adolphe Robert, enfin, publia un annuaire des proscrits du Deux Décembre ${ }^{42}$ et collabora plus tard au Dictionnaire Historique et Biographique de la Révolution et de l'Empire 43 dirigé par Robinet. En poussant l'investigation du coté des collaborateurs de ce dictionnaire on trouve tout un personnel partageant des dispositions sociales et politiques analogues à celles de nos trois auteurs ${ }^{44}$. Pour sa part, le docteur Robinet, conservateur du musée Carnavalet et exécuteur testamentaire d'Auguste Comte présidait la Société positiviste. Il était spécialiste de Danton et entretenait des relations avec Alphonse Aulard dont il partageait l'intérêt pour ce personnage révolutionnaire dans le cadre de la Société d'histoire de la Révolution française ${ }^{45}$. L'engagement républicain et l'œuvre d'Alphonse Aulard sont suffisamment connus ${ }^{46}$; signalons seulement que la chaire d'histoire de la Révolution française créée à son intention en Sorbonne par la ville de Paris en 1886 lui permit d'influer sur l'interprétation républicaine de la Révolution. Comme on le voit, ces relations plaçaient socialement et intellectuellement nos auteurs au cœur des enjeux commémoratifs du centenaire de la Révolution.

Ce bref portrait de groupe laisse entrevoir la formation intellectuelle et l'engagement politique de ces hommes dont la combinaison de positions secondaires dans l'enseignement, le barreau, les sociétés savantes, l'édition et

41. Les archives municipales montrent que Cougny tenta d'être élu maire mais n'obtint qu'une voix au sein du conseil municipal! Il fut cependant très impliqué et novateur dans la gestion des affaires scolaires de la ville de Bourges en imposant non seulement la gratuité scolaire totale mais aussi en proposant d'étendre la diffusion culturelle en direction des classes laborieuses par l'ouverture des lieux culturels aux classes populaires.

42. Adolphe Robert, Statistique pour servir à l'histoire du 2 décembre 1851. Paris et les départements, Paris, Librairie de la Renaissance, 1869. L'ouvrage ne manque pas de surprendre puisqu'il ne comporte qu'une liste nominative des proscrits sans aucun commentaire général ni aucune information biographique! Malgré ce dépouillement, on peut prêter à cet ouvrage paru en 1869 une portée critique certaine.

43. Dictionnaire Historique et Biographique de la Révolution et de l'Empire, 1898.

44. Cet ouvrage a réuni 18 collaborateurs dont certains sont "avocats en titre» (nos recherches aux archives de l'Ordre des avocats ayant révélé une faible activité), d'autres publicistes, enseignants ou anciens députés (Baudin, Charles Laisant et Massé). Certains ont un passé contestataire très marqué (Winant très lié à la Commune ou Charles Laisant qui, venant de l'extrême gauche, rejoignit le camp boulangiste).

45. En 1893, la composition du bureau de cette société était la suivante : Sadi Carnot en était le président d'honneur, Jules Claretie le président en exercice, Robinet, Parfait et Guiffrey (archives nationales) se partageaient la vice-présidence tandis que Alphonse Aulard en assurait le secrétariat général.

46. Les références à Alphonse Aulard sont nombreuses, compte tenu de son rôle charnière dans l'historiographie révolutionnaire et des controverses dont son œuvre fit les frais après la première guerre mondiale. Pour un bref aperçu sur ce personnage, voir Christophe Charle, Les élites de la République, 1880-1900, Paris, Fayard, p. 247. 
la politique tirait sa possibilité de la construction d'une «société parlementaire» dans laquelle ils purent convertir leurs dispositions. Les positions qu' ils occupaient dans cette formation sociale peuvent paraître marginales mais, à la faveur de la conjoncture des années 1880 , elles les prédisposèrent à prendre part - et parti - dans les «luttes d'autonomisation» de l'activité parlementaire et, plus particulièrement, dans les «luttes biographiques». Tout indique donc que l'on ne peut comprendre le sens de ce travail en le rapportant seulement aux dispositions des auteurs car leur entreprise était liée aux offres de typification du métier parlementaire et aux enjeux issus du champ politique.

\section{LES CONDITIONS POLITIQUES ET INTELLECTUELLES D'ÉCRITURE DU DICTIONNAIRE DES PARLEMENTAIRES}

Loin d'être un ouvrage d'historiens, le Dictionnaire des parlementaires manifeste l'implication, périphérique mais bien réelle, de ses auteurs dans les enjeux d'un espace parlementaire duquel leur entreprise d'objectivation tire une grande part de ses raisons d'être et de ses moyens matériels et cognitifs. Qu'il s'agisse du moment, de la méthode, des usages pratiques auxquels il était destiné ou des catégories d'identification qui ont présidé à sa réalisation, le Dictionnaire des parlementaires résulte d'une rencontre de ses auteurs avec les enjeux propres à l'univers parlementaire.

Pour ce qui concerne le moment, le premier signe de cette dépendance réside dans la conjoncture politique des années 1880 qui détermina la rédaction du dictionnaire. Les trois auteurs étaient liés, à un titre ou à un autre, à la municipalité parisienne alors très à gauche, et cette position, même modeste, les plaçait au cœur de l'enjeu politique constitué autour de la commémoration de 1789. De même, leur rapport avec la Société d'histoire de la Révolution fut déterminant puisque le Dictionnaire des parlementaires semble justifié et inspiré, y compris dans l'agencement des fiches biographiques, par l'historiographie républicaine. Les enjeux que le centenaire de la Révolution suscita dans le camp républicain ${ }^{47}$ dès le milieu des années 1880 produisirent une structure d'opportunité favorable à ces hommes qui se trouvèrent alors en mesure de valoriser leurs compétences et d'obtenir des soutiens municipaux puis parlementaires. Le Dictionnaire des parlementaires n'est d'ailleurs qu'un exemple parmi les nombreux projets commémoratifs proposés aux comités républicains par les associations de commémoration de 1789 (essentiellement l'Association républicaine du centenaire de 1789, opportuniste, et la Fédération

47. La commémoration suscite la création de deux associations républicaines, l'Association républicaine du centenaire de 1789, opportuniste, et la Fédération de 1889, radicale. Pour une analyse de la concurrence républicaine autour du centenaire, on pourra se reporter à Raymond Huard, «Le centenaire de 1789 et les origines du parti radical, la Fédération de 1889", dans Le XIXe siècle de la Révolution française, Paris, Créaphis, 1992, p. 129-148. 
de 1889, radicale). Cet engouement des républicains pour la Révolution faisait suite à la frayeur que leur avaient causée les élections législatives de 1885 . Dès lors, beaucoup d'entre eux, dont bon nombre de parlementaires ${ }^{48}$, virent dans cette commémoration un moyen parmi d'autres de renforcer leur organisation et de préciser leur doctrine ${ }^{49}$. La logique commémorative était donc prise dans une concurrence interne au camp républicain pour l'appropriation politique de l'héritage révolutionnaire. Le travail d'unification séculaire des parlementaires réalisé par le "Robert et Cougny» résulta directement de cet enjeu de mémoire qui transforma le souvenir révolutionnaire en une opération d'anamnèse prenant le moment révolutionnaire pour origine de la plupart des institutions et des engagements républicains du moment. C'est en effet dans la conjoncture politique des années 1880 avec ses enjeux et ses catégories que se définit "l'horizon de rétention " ${ }^{50}$ depuis lequel, en remontant jusqu’à la Révolution, tous les parlementaires avec leurs propriétés et leurs accomplissements particuliers, furent saisis et enrôlés dans un temps et un horizon d'action qui n'étaient pas les leurs ${ }^{51}$. En rassemblant sous un même substantif et selon des attendus semblables des personnages que leur activité et leur statut séparaient, le Dictionnaire des parlementaires réalisa une sorte de coup de force symbolique par lequel il «inventa», littéralement, les parlementaires. Il va de soi que cette mobilisation du passé était en accord avec le travail de consolidation d'un régime qui, défini autour du parlementarisme, avait tout à gagner dans «l'éternisation" et l'universalisation de la fonction parlementaire réalisée par ce dictionnaire. Conçue durant la crise boulangiste, cette entreprise d'unification symbolique de la fonction parlementaire pouvait passer alors pour une ligne de défense contre l'antiparlementarisme. Par ailleurs, la distance critique du Dictionnaire des parlementaires vis-à-vis des députés du Second Empire ${ }^{52}$, la valorisation de certains parcours militants d'extrême gauche ou encore le choix des votes significatifs de la dernière législature (réforme constitutionnelle, loi sur la presse, poursuites contre Boulanger, expulsion des princes, soutien à Rouvier, etc.) attestent également de l'engagement des auteurs dans une époque où leur ouvrage devait jouer un rôle.

Concernant la méthode, l'insertion des auteurs dans l'espace de production de l'image publique des parlementaires se traduisit pratiquement, pour la collecte comme pour l'agencement des données, par un dispositif de travail

48. Une centaine de parlementaires participèrent directement à la commémoration du centenaire. 49. Après l'avertissement des élections de 1885 , les républicains tentèrent de fédérer les comités. Rappelons que cette tâche, confiée à Jules Steeg en 1886, déboucha en 1887 sur un congrès rassemblant environ 250 comités.

50. Notion empruntée à Maurice Merleau-Ponty, Phénoménologie de la perception, collection Tel, Paris, Gallimard, 1992, p. 476 et s.

51. Pour un aperçu sur les différences historiques d'acception du rôle parlementaire, on pourra se reporter à Jean Joana, Pratiques politiques des députés français au XIXé siècle, Du dilettante au spécialiste, Paris, L'Harmattan, 1999, 311 p.

52. C'est à juste titre que Éric Anceau déplore le traitement réservé aux députés du Corps législatif par le "Robert \& Cougny». Voir Éric Anceau, Dictionnaire des députés du Second Empire, Presses Universitaires de Rennes, 1999, 421 p. 
articulant leurs routines intellectuelles préexistantes à une offre institutionnelle d'identification des parlementaires à laquelle les exposait leur collaboration avec les assemblées représentatives. Cette tension entre les procédés attachés aux exercices savants auxquels ils étaient accoutumés et les catégories indigènes s'imposant à eux déboucha sur une méthode assez éclectique dont le dictionnaire porte la trace.

Ces professionnels de l'écriture possédaient, pour deux d'entre eux tout au moins, une expérience en matière biographique. De sorte que leur dispositif de travail avec ses routines, ses stocks de données biographiques, ses réseaux d'échange, ses principes d'agencement, etc. existait antérieurement et indépendamment de l'entreprise éditoriale qu'il orienta. Coutumiers de ce type de recherche, les auteurs vérifièrent l'état civil des représentants, recueillirent des informations biographiques à la Bibliothèque Nationale, aux Archives Nationales, etc. Robert poursuivit le travail entrepris au même moment avec Robinet pour le Dictionnaire de la Révolution et de l'Empire. Bourloton, quant à lui, recycla les données et savoir-faire constitués en d'autres circonstances: ainsi fut-il conduit à activer un réseau d'érudits provinciaux avec lesquels il échangea des informations biographiques, comme en attestent de nombreuses correspondances ${ }^{53}$. Ce travail de collecte reposait donc sur l'antériorité d'une économie des données biographiques composée d'informations recueillies dans les annuaires précédents, la presse, les biographies régionales, les nobiliaires, les almanachs départementaux, les archives familiales, etc. Cet éclectisme des sources transparaît au détour de certaines fiches chargées d'indications familiales ou amicales et d'anecdotes personnelles qui mettent à mal l'unité taxinomique de l'entreprise.

Cougny semble s'être consacré au dépouillement des archives parlementaires dont il convient de souligner la portée particulière dans ce dictionnaire. Les affinités nouées avec les élus et le personnel des assemblées dans le cadre du centenaire de 1789 expliquent ce recours aux archives parlementaires et aux tables analytiques nominatives des assemblées ${ }^{54}$ qui introduisirent une nouveauté car, s'ils relataient l'activité parlementaire des élus, les dictionnaires antérieurs ne lui accordaient pas une telle place. Ici encore, le moment de rédaction du «Robert et Cougny» est décisif car il correspond au déploiement d'une bureaucratie parlementaire tournée vers la mise en forme des débats,

53. Par exemple, nous avons pu retrouver quelques courriers qui témoignent de ses échanges avec Jean Delmas, un érudit du Cantal auprès duquel il recueille des informations sur les parlementaires du Cantal (Archives Départementales du Cantal, fonds Jean Delmas, dossier $n^{\circ} 81$ ). Les autres cartons du fonds Delmas contiennent toutes sortes de données biographiques et de coupures de presse concernant les parlementaires cantaliens qui donnent la mesure de l'éclectisme des sources du «Robert et Cougny".

54. Les auteurs consultèrent non seulement les tables mais aussi les déclarations d'état civil remplies par les nouveaux élus que nous analysons plus loin. Rappelons que le travail parlementaire de chaque session est regroupé dans des tables analytiques nominatives et par matière. Elles permettent de se retracer aussi bien l'activité d'un parlementaire que le cheminement d'une loi et elles renvoient aux volumes impressions et annales dans lesquels on retrouve, respectivement, les textes et les débats parlementaires. 
des discours, des rapports des commissions et même vers l'édition des annales de la période révolutionnaire ${ }^{55}$. Par cette référence à l'offre d'objectivation indigène, qui annonce l'imbrication de ses successeurs dans l'activité bureaucratique des assemblées, le Dictionnaire des parlementaires prenait acte d'un type de spécialisation de l'activité parlementaire autour de laquelle il bâtit une nouvelle figure des parlementaires. La mention systématique du travail parlementaire (tel qu'il était appréhendé par les tables) renouvela l'identification d'une population dont elle facilitait l'unification en donnant l'illusion de la permanence de son activité et en renvoyant les variations synchroniques et diachroniques de l'endossement du rôle parlementaire dans le foisonnement des histoires individuelles. Dans le contexte de la crise boulangiste, cette identification possédait la vertu non négligeable d'éloigner les stigmatisations négatives attachées à d'autres catégories d'entendement comme celle de politicien, terme importé à la même époque ${ }^{56}$. Le Dictionnaire des parlementaires differe donc de ses devanciers comme de ses successeurs quant à l'état du champ de représentation dans lequel il s'inscrit car, contrairement aux premiers, sa confection s'appuie déjà sur des taxinomies parlementaires sans être encore, à l'inverse des seconds, totalement imbriquée dans le travail bureaucratique des assemblées.

À l'instar des annuaires professionnels, le Dictionnaire des parlementaires revendiquait également une portée pratique : mieux connaître untel, son passé, ses mandats antérieurs, ses conditions d'élection, son ancienneté, ses compétences, ses prises de position sur les grands votes, etc. Autant d'informations qui plaçaient les dictionnaires dans l'horizon d'action des députés, des publicistes, etc. Bourloton comptait certainement sur ces usages indigènes pour la réussite éditoriale de l'ouvrage; en tout cas, il ne pouvait pas les ignorer. Ce souci, commun aux annuaires homologues, s'est traduit par le soin apporté à la transcription des résultats électoraux, des votes à la Chambre, etc. Mais, dans l'esprit de ses concepteurs, le Dictionnaire des parlementaires revêtait par-dessus tout une vertu pédagogique et démocratique qui nous échappe si l'on oublie la proximité des tentatives de rationalisation de l'opinion dont on trouve un exemple dans le "Barodet" qui, dès 1885, glanait les professions de foi des candidats ${ }^{57}$. Bourloton partageait l'opinion encore commune concernant la nocivité des luttes partisanes et, loin d'avoir conscience de prendre part à l'autonomisation du travail de représentation, il estimait que l'utilité de son œuvre "n'avait pas besoin d'être démontrée dans

55. Hervé Fayat, "Le métier parlementaire et sa bureaucratie», dans Guillaume Courty [dir.], Le travail de collaboration avec les élus, Paris, Michel Houdiard, 2005, p. 29-48. Les années 1880 connurent un développement des services législatifs, particulièrement du service de la présidence dirigé par Eugène Pierre. Les anciennes annales ont été reconstituées durant ces années et ce travail représenta un effort considérable pour les services parlementaires.

56. Dominique Damamme, "Professionnel de la politique, un métier peu avouable», dans Michel Offerlé [dir.], La profession politique, XIXe XXe siècles, Paris, Belin, 1999, p. 37-67.

57. Sur cet aspect de la rationalisation du vote, voir Alain Garrigou, Le vote et la vertu, Paris, Fondation nationale des sciences politiques, $1992,288 \mathrm{p}$. 
un pays de suffrage universel» et qu'elle contribuait aux «luttes fécondes des doctrines et des idées, qui sont le ressort le plus actif du progrès social, le profit direct et l'honneur même de la liberté». Ces remarques doivent inviter l'historien à tenir compte des objectifs pratiques comme des usages autochtones de ces ouvrages lorsqu'il en fait la source du travail historiographique.

La représentation des parlementaires émanant du «Robert et Cougny» est enfin tributaire des perceptions et classifications sociales mobilisées par ses auteurs et, en particulier, de leur rapport aux offres sociales de typification du «métier parlementaire», c'est-à-dire aux identifications dont la consistance tient à la capacité de certains groupes à changer leur identité sociale en marque politique légitime : notables, fonctionnaires, professeurs, avocats, militants et militaires ${ }^{58}$, etc. Ces "marques» sont simultanément des moyens d'enregistrement des propriétés sociales des parlementaires et des instruments de représentation politique ${ }^{59}$ utilisés par les candidats. Activant des enjeux aussi bien politiques (les «nouvelles couches» de Gambetta) que savants ${ }^{60}$ (voir le portrait du politicien comme «déclassé» chez Ostrogorski ou comme «entrepreneur» chez Weber, etc.), l'usage de ces représentations pilote l'agencement des matériaux biographiques (amas de propriétés disparates ou ambiguës, d'occurrences désordonnées et d'engagements de circonstance...) mais, du même coup, l'utilisation de ces topiques ratifie les principes de délégation sociale et politique desquels ils sont issues. Le rapport d'usage de nos auteurs à l'égard de ces catégories de représentation exprime sans surprise leur propre distance sociale vis-à-vis des groupes en compétition pour les positions électives et livre leur point de vue sur la légitimité des représentants. Par exemple, ils projettent sur le monde politique certains enjeux et principes de classement académiques et esthétiques dont témoigne la valorisation des titres scolaires et des œuvres; le cursus scolaire est soigneusement détaillé et accompagné de mentions telles que "solides études» ou "brillantes études", "secrétaire de la conférence du stage» pour les avocats, de même qu'ils recensaient scrupuleusement les ouvrages publiés ou les conférences prononcées en fin de fiche. Significative de leur expérience sociale, cette affinité du titre et du poste les conduisit à promouvoir la figure du «savant républicain» et à substituer, non sans un certain réalisme historique, la légitimité scolaire à "l'autorité sociale évidente» des notables, selon la formule de André Siegfried. Dans le même ordre d'idées, certaines fiches s'écartent du destin politique du représentant pour se concentrer sur ses œuvres au point de se changer en article de critique littéraire. De même faut-il mettre sur le compte de leur

58. Laurent Willemez, «La République des avocats, le mythe, le modèle et son endossement» dans, La profession politique XIX'e-XXe siècle, ouv. cité, p. 201-229.

59. Sur cette ambivalence de la notion de représentation, voir Alain Desrosières et Laurent Thévenot, Les catégories socio-professionnelles, collection Repères, Paris, La Découverte, $4^{\mathrm{e}}$ édition, 2000, 121 p.

60. Rien ne nous permet de prêter aux constructions savantes qui paraissent à cette époque dans la Revue de l'école libre des sciences politiques une quelconque influence sur les auteurs du Dictionnaire des parlementaires. 
inclination politique une légitimation des parcours militants souvent exposés avec emphase surtout, bien sûr, s'il s'agit d'opposants au Second Empire. À ces formes de sympathie s'ajoutent des marques d'empathie jalonnant le récit de vie : participation à la défense nationale, voyages, exil...

Contrairement à ses successeurs, le "Robert et Cougny» ne livre donc pas, à proprement parler, des biographies d'institution ${ }^{61}$ car, à l'inverse de ces dernières qui réduisent le complexe de personnages sociaux que recèle chaque individu concret à quelques figures stylisées, il utilise des catégories de présentation des parlementaires relativement hétérogènes, comme le suggèrent les méthodes et principes évoqués ci-dessus. Mais, soutenu par des dispositifs institutionnels d'enregistrement de l'activité parlementaire, structuré par des enjeux politiques, orienté par l'historiographie républicaine, tendu vers l'unification de pratiques et d'accomplissement divergents et enfin, appelé par l'usage à façonner les modalités de présentation de soi dans l'univers parlementaire, le Dictionnaire des parlementaires constitue une sorte «d'illusion bien fondée» dont atteste la pérennité tant sociale que savante du point de vue qu'il initia. Par tous ces traits, le "Robert et Cougny» est souvent perçu comme l'attestation d'une institution parlementaire réalisée. Il s'agit donc maintenant de décrire la façon dont les auteurs sont parvenus à faire endosser à des individus concrets le personnage social composite qu'ils ont créé.

\section{LA CONSTRUCTION D'UN DÉPUTÉ DE PAPIER}

La tentation est grande de voir dans les fiches du «Robert et Cougny» des totalisations rétrospectives d'expériences, de positions et de prises de positions éparses dans le temps comme dans l'espace social, n'ayant guère plus d'unité que le patronyme sous lequel ces divers rôles sociaux ont été accomplis, ni d'autre principe d'assemblage que la prise de position des auteurs dans les "luttes d'autonomisation" d'un espace parlementaire ${ }^{62}$. La contingence des fiches biographiques à l'égard de leurs auteurs, de leur époque et de leurs conditions sociales et intellectuelles d'engendrement doit-elle conduire à renvoyer ces processions de parlementaires de papier au néant des artefacts? Non, car cette série de portraits relève, à y regarder de plus près, d'un travail d'institution d'une carrière parlementaire qu'illustrent ces parcours de vie orientés vers l'accès à la députation. Arrachées parfois à leur époque et souvent à leur temps propre, les propriétés, actions et occurrences individuelles de ces "députés en gésine» sont massivement rabattues sur un temps et un espace politiques auxquels elles n'appartiennent pas d'emblée. La courbure

61. Voir Éric Agrikoliansky, art. cité.

62. Elles suggèrent des remarques semblables à celles que Pierre Bourdieu formulait à propos du genre biographique et des récits de vie en général. Voir, Pierre Bourdieu, «L'illusion biographique», dans Actes de la recherche en sciences sociales, $\mathrm{n}^{\circ}$ 62-63, juin 1986, p. 69-72. 
du temps et de l'espace social que cet exercice fait subir aux parcours individuels se comprend mieux si on la rapporte à l'enjeu implicite qui «travaille» les biographies : l'autonomisation d'un espace parlementaire pourvu d'un temps propre, de rythmes singuliers et de carrières avec leur cursus honorum. Dès lors, le Dictionnaire des parlementaires apparait comme une sorte de matrice qui, procédant à une manipulation du temps - durée et tempo - et de l'espace social - étendue et structure - intègre les biographies dans une structure narrative commune (les fiches sont plus ou moins standardisées) dessinant les contours d'une carrière parlementaire.

Concernant tout d'abord le temps, le Dictionnaire des parlementaires réalise une sorte de "synthèse temporelle " ${ }^{63}$ par laquelle les occurrences personnelles sont annexées à des séquences historiques relevant d'une organisation rétrospective du flux d'événements politiques, cette organisation étant plus ou moins redevable à l'historiographie républicaine. Selon ce principe, chaque cohorte de députés est confrontée aux mêmes événements contemporains et les prises de position individuelles ou familiales sont rapportées à ce temps supposé public. La structure de ce temps factice précipite les représentants, par une curieuse anticipation des effets de détermination et de synchronisation que l'univers parlementaire impose à ses ressortissants, dans un temps social et dans un tempo politique commun avant même leur premier mandat. Les individus ne sont certes pas réduits à une existence purement nominale, mais leur vie est rapportée à un espace spécialisé plutôt que restituée dans son déroulement intime car, pour les auteurs comme pour leurs lecteurs, seules comptent les propriétés et occurrences "pertinentes» ${ }^{64}$.

C'est une inflexion d'un autre ordre que nous observons concernant l'indexation des propriétés sociales. De nos jours, la nomenclature des catégories socio-professionnelles s'impose à la plupart des opérations de classement social, de sorte que, la structure des positions sociales étant plus ou moins admise, les observations critiques peuvent se concentrer sur le relevé des propriétés sociales ou la distribution des populations. Ce n'était évidemment pas le cas à la fin du XIX siècle où chaque opération de classement social véhiculait des principes classificatoires implicites, eux-mêmes en jeu dans ce travail de classement. C'est pourquoi à cette époque de concurrence des principes de représentation de l'univers social, ces documents sont tout aussi instructifs du point de vue des catégories qu'ils utilisent que des informations sociographiques qu'ils contiennent ${ }^{65}$. Aussi, bien qu'il s'engendre à l'insu de ses

63. Norbert Elias, Du temps, Paris, Fayard, 1996, p. 140. Faute de pouvoir préciser ici la portée et les conditions historiques et intellectuelles de cette synthèse temporelle, signalons seulement qu'elle est ce par quoi les auteurs attribuent à tous les parlementaires une même perception du flux des événements et, au-delà, une même temporalité.

64. Sur cette tension entre la structure et l'individu dans les récits de vie, on pourra consulter Dominique Dammame, "Grandes illusions et récits de vie», dans Politix, 1992, n²7, p. 183-188.

65. L'indécision dans laquelle se trouve alors la statistique quant au classement selon le secteur d'activité, la place dans les rapports de production ou le métier laisse entrevoir la divergence des principes de classement social. Voir Alain Desrosières, Laurent Thévenot, Les catégories socio-professionnelles, ouv. cité. 
auteurs et au fil de leur collecte d'informations, le système de représentation sous-jacent au Dictionnaire des parlementaires n'en exprime pas moins une vision de la distribution sociale des «chances typiques" d'accès et de succès dans la carrière parlementaire et, surtout, une prise de position dans les luttes de définition des propriétés sociales légitimes et/ou pertinentes en politique; certaines des positions évoquées plus haut telles que avocat, médecin ou professeur fonctionnent clairement comme des "marques politiques" positives pour les auteurs auxquels elles évoquent la méritocratie, l'engagement, le dévouement, la rationalité, etc.

Afin de rendre cette vision plus tangible, nous avons effectué un relevé exhaustif et un classement ${ }^{66}$ de tous les attributs retenus par le «Robert et Cougny" pour caractériser les députés accédant à leur premier mandat durant la législature contemporaine de l'écriture du dictionnaire (soit 299 députés pour la législature 1885-1889). Cet examen - exposé ici au niveau le plus agrégé - révèle une prédilection des auteurs pour les propriétés les plus proches de... leur propre position! Si les informations relatives à l'inscription dans le champ politique (39\% des positions cumulées et $55 \%$ des députés) arrivent en tête, il faut signaler que la moitié de ces attributs expriment tantôt une activité «militante» ou contestataire (exil ou proscription, participation à un comité, à un congrès international...), tantôt une position parlementaire remarquable (rapporteur de commission, interpellation remarquée ou dépôt d'une proposition...). De sorte que les positions électives locales forment une assez faible part des attributs biographiques, à peine plus que les titres scolaires ( $12,7 \%$ des positions cumulées mais $55 \%$ des députés) qui, chronologie oblige, se trouvent en début de fiche aux cotés des origines familiales (on signale surtout la présence d'ascendants ou de collatéraux députés dans $18 \%$ des cas!) à l'inverse des titres honorifiques et patrimoniaux qui clôturent la fiche (6,3\% des positions cumulées). Pour ce qui concerne les positions professionnelles, on retiendra une domination sans surprise des professions juridiques et essentiellement des avocats en titre ou en exercice, qualité généreusement accordée par les auteurs dont deux d'entre eux pouvaient revendiquer ce titre $(28 \%$ des députés sont crédités de cette profession, chiffre placé dans la fourchette des enquêtes prosopographiques ${ }^{67}$ ) mais, finalement, cette propriété est relativisée puisqu'elle représente seulement $6,7 \%$ du total des positions attribuées à l'effectif $(9,3 \%$ pour l'ensemble des professions juridiques). On relèvera également une propension à faire apparaître un passé

66. Pour la réalisation de ce tableau, nous nous sommes inspirés de Luc Boltanski, «L’espace positionnel, multiplicité des positions institutionnelles et habitus de classe», dans Revue française de sociologie, janvier-mars 1973, p. 3-26. La transposition ne visait pas tant à révéler la multi-positionnalité des députés (cela aurait exigé une enquête individuelle) qu’à mettre au jour l'usage politique des identités sociales par les députés.

67. Les sources sont nombreuses, voir, entre autres, Gilles Le Béguec, «Prélude à la République des avocats", dans Alain Corbin, Jean Marie Mayeur et Arlette Schweitz [dir.], Les immortels du Sénat, Paris, Publications de la Sorbonne, 1994. 
patriote ( $13 \%$ des députés sont considérés comme volontaires en 1870) ou un grade dans l'Armée si bien qu'un quart des représentants se trouve lié, à un titre ou à un autre, à l'univers militaire. La tendance à valoriser le travail intellectuel et artistique conduit par ailleurs les auteurs à recenser largement les publications ou les collaborations éditoriales si bien que bon nombre de députés sont revêtus d'un statut d'essayiste ou de publiciste qu'ils ne revendiqueraient pas eux-mêmes (5\% des positions cumulées mais $22 \%$ de l'effectif). On l'aura compris, à suivre les auteurs dans cette énumération de propriétés et d'occurrences "pertinentes", on s'éloigne certainement des conditions concrètes d'accumulation du capital politique, mais on se rapproche de ses justifications.

Envisagées du point de vue de leur étendue et de leur structure, les catégories d'identification employées par le Dictionnaire des parlementaires pour cette législature permettent donc de formuler quelques remarques valables pour l'ensemble du travail d'objectivation sociale conduit par ses auteurs. On relèvera tout d'abord l'amplitude sociale considérable des positions relatées qui, inhérente à l'accumulation de positions successivement occupées au cours d'une vie, est encore amplifiée par la perception extensive qu'ont les auteurs des formes de l'activité politique. Mais, comme le notait Ernest Labrousse pour caractériser le passage des ordres aux classes sociales ${ }^{68}$, la production de classements sociaux tient moins aux critères eux-mêmes qu'à leur combinaison.

De ce point de vue, la conception que les auteurs se font du cycle de vie génère une séquence typique (héritage - formation - profession - entrée en politique - candidature - carrière et prise de position parlementaire - publications et titres honorifiques éventuels) qui impose d'elle-même une hiérarchie des trophées et une succession des accomplissements dans lesquels le lecteur doit percevoir une montée en puissance de la «vocation». Au premier rang de ces accomplissements figurent les titres scolaires. Bien que reconnue par l'historiographie ${ }^{69}$, cette importance accordée aux trophées scolaires exprime bien plus la foi méritocratique des auteurs que la réalité politique, mais le crédit des parlementaires se trouve renforcé par cette correspondance du titre et du poste. Cependant, l'originalité de la "gestion symbolique» que propose le Dictionnaire des parlementaires réside moins dans la hiérarchie des légitimités sociales que dans le système d'opposition qu'il suppose. S'il met en scène les oppositions politiques de son époque (du type républicains/bonapartistes), le Dictionnaire des parlementaires expose aussi des clivages spécifiques à l'univers parlementaire (orateur/non orateur, expert/non expert, etc.) et des principes de division du champ politique (méritocratie/héritage, militantisme/notabi-

68. Ernest Labrousse, "Conclusion", dans Ordres et classes. Colloque d'histoire sociale, Saint-Cloud, 24-25 mai 1967, Paris - La Haye, 1973, p. 267 et s.

69. Christophe Charle, "Les parlementaires : avant-garde ou arrière-garde d'une société en mouvement?», dans Les parlementaires de la Troisième République, ouv. cité, p. 51 et s. 
lité, univers intellectuel/univers économique, etc...) qui survivront à cette époque ${ }^{70}$. La pérennité de ces principes de division explique la longévité de cet ouvrage car ils ouvrent un espace positionnel dans lequel on pourra inscrire de multiples positions et tracer de nombreuses figures politiques.

Afin de mettre en valeur l'arbitraire classificatoire du "Robert et Cougny" et de montrer la variété des principes de présentation applicables à une même population, nous avons renouvelé l'opération précédente avec les mêmes députés en partant, cette fois, des déclarations d'état civil qu'ils remplissaient lors de leur première élection ${ }^{71}$. Ces propriétés sociales "spontanées» ont ensuite été classées dans les mêmes champs en consignant leur rang d'énonciation par une numérotation. Ce test permet de mesurer l'ampleur des écarts entre le personnage social construit par le Dictionnaire des parlementaires et celui qui découle de l'agrégation des stratégies indigènes de présentation de soi. Or, les coefficients de corrélation significatifs ou complets (c'est à dire compris entre 0,5 et 1 ) des deux séries ne concernent qu'un quart à peine des élus; autant dire que les séries sont quasiment indépendantes. L'essentiel n'est pas dans ce résultat attendu mais dans la divergence des principes de production des identités selon qu'elles proviennent de députés enclins à y voir des biens de représentation ou des auteurs du Dictionnaire des parlementaires qui, moins concernés par cet usage politique de la position sociale, projettent dans cette opération d'objectivation sociale les enjeux propres à leur milieu.

Lors de leur premier contact avec la Chambre, les députés, saisis par la logique légitimiste de la prise de fonctions, ont des réflexes parfois surprenants (pour seule qualité sociale, Jaurès déclare «célibataire»!) mais, dans leur immense majorité, ils font montre de stratégies de présentation de soi tout à fait lisibles. Tout d'abord, l'éventail des positions citées est logiquement plus restreint socialement et temporellement que celui du "Robert et Cougny" car il parcourt généralement une portion plus restreinte du cycle de vie et, de plus, il se concentre sur les identités jugées «stratégiques" par les élus; en contrepartie, il fait apparaittre une dimension essentielle que dissimulent les dictionnaires biographiques : la multi-positionnalité des parlementaires. Ainsi, la distribution des identités sociales est bien différente; la part des positions juridiques est plus forte $(16,6 \%$ du total des positions occupées par l'effectif au lieu de $9 \%$ ) et concerne plus du tiers des députés alors qu'un dixième seulement des élus déclarent un titre scolaire et qu'un quart d'entre eux font état d'un titre honorifique.

Ensuite, l'ordre de déclaration des positions évoque une configuration des rapports de force sociaux (entre les diverses «marques politiques» évo-

70. Même dépassées dans la pratique comme le montre Éric Phélippeau à propos du baron de Mackau, ces oppositions un peu simplistes demeurent des catégories d'appréhension communes; voir Éric Phélippeau, Linvention de l'homme politique moderne, Paris, Belin, 1999, 352 p.

71. Ces registres manuscrits sont conservés aux archives de l'Assemblée Nationale et s'en tiennent à l'état civil des élus. 
quées plus haut) qu'occultent les dictionnaires parlementaires centrés sur la chronologie et que nous nous proposons d'illustrer par l'exemple des députés possédant l'attribut «avocat».

\section{LES DÉCLARATIONS SPONTANÉES DES DÉPUTÉS POSSÉDANT L'ATTRIBUT «AVOCAT »}

\begin{tabular}{|c|c|c|c|c|c|c|}
\hline & & $\begin{array}{l}\text { ang de d } \\
\text { l'attribu }\end{array}$ & $\begin{array}{l}\text { léclarati } \\
\text { it «avoc }\end{array}$ & & & \\
\hline $\begin{array}{l}\text { nombre de positions } \\
\text { déclarées dont celle } \\
\text { d'avocat }\end{array}$ & rang 1 & rang 2 & $\operatorname{rang} 3$ & $\operatorname{rang} 4$ & $\begin{array}{c}\text { Cumul des } \\
\text { positions }\end{array}$ & $\begin{array}{c}\text { Nombre } \\
\text { de positions } \\
\text { en } \% \text { cumulés }\end{array}$ \\
\hline 1 & 23 & & & & 23 & $35,94 \%$ \\
\hline 2 & 17 & 6 & & & 69 & $53 \%$ \\
\hline 3 & 8 & 0 & 3 & & 102 & $78 \%$ \\
\hline 4 & 3 & 1 & 1 & 1 & 126 & $96 \%$ \\
\hline 5 & & 1 & & & 131 & $100 \%$ \\
\hline $\begin{array}{l}\text { total par rang de } \\
\text { déclaration }\end{array}$ & 51 & 8 & 4 & 1 & 64 & \\
\hline $\begin{array}{l}\text { Effectifs par rang } \\
\text { en \% cumulés }\end{array}$ & $79,7 \%$ & $92,2 \%$ & $98,4 \%$ & $100 \%$ & 2,04687 & \\
\hline
\end{tabular}

La lecture du tableau ci-dessus nous montre l'exceptionnel usage symbolique de l'identité d'avocat par les nouveaux députés. On y relève tout d'abord un "effet d'attraction" puisque, sur 64 députés susceptibles de revendiquer cette position (avocats en titre ou en exercice), 51 (soit $80 \%$ d'entre eux) font état de cette qualité au premier rang de leur déclaration et 59 (soit 92\%) aux deux premiers rangs. On remarque ensuite un «effet de substitution" puisque la plupart de ceux qui placent cette identité au premier rang n'éprouvent pas le besoin de mentionner une autre occupation ou un mandat électif (du coup, ils ne déclarent que deux positions en moyenne, 2,046 soit 131/64). Ces deux principes de production identitaires traduisent respectivement les caractères "d'identité stratégique ${ }^{72}$ » et "d'identité métonymique» associés à l'avocature qui, pouvant être utilement produite dans la plupart des occasions politiques, constitue aussi une "identité ubiquitaire». Ces trois caractéristiques font de cette «marque» sociale une «identité dominante» dans le champ parlementaire de cette époque ${ }^{73}$, cette préférence étant encore plus marquée pour les

72. Sur cette notion, voir Annie Collovald, «Identités stratégiques», dans Actes de la recherche en sciences sociales, $\mathrm{n}^{\circ} 73$, juin 1988 , p. 29-40.

73. Par le terme "d'identité dominante» nous entendons seulement signaler que les identités sociales sont hiérarchisées par l'univers politiques dans lequel elles sont produites. 
classes d'âge les plus jeunes ${ }^{74}$. Aucune autre identité sociale ne se prête à un usage symbolique aussi intense, comme le montrent les tableaux semblables réalisés pour les députés possédant des attributs d'une fréquence comparable tels que "maire» ou "conseiller général » ${ }^{75}$. Bien sûr, rien de tel n'apparaît dans le «Robert et Cougny» qui introduit «chrono-logiquement» la position d'avocat au second rang après la formation juridique (le groupe modal des députés possédant cet attribut correspond au second rang et représente $43 \%$ des effectifs susceptibles de revendiquer cette qualité).

Sans poursuivre plus avant dans la divergence des principes de présentation de soi que cette comparaison laisse entrevoir, on peut mesurer tout le profit que l'historiographie pourrait tirer de la prise en compte de l'usage politique des identités sociales, en particulier par l'introduction d'une analyse configurationnelle qui complèterait utilement l'analyse longitudinale qui prévaut habituellement.

\section{LA RÉUSSITE SOCIALE ET SAVANTE D'UNE «ILLUSION BIEN FONDÉE "}

La réussite sociale $\mathrm{du}$ "Robert et Cougny» repose d'abord sur l'intérêt pratique des parlementaires pour un ouvrage qui permet de mieux connaître untel, sa circonscription, etc. Son crédit, à l'instar des annuaires professionnels, repose partiellement sur cette inscription passée dans la trame des relations parlementaires. Cette fonction s'est renforcée, à compter de 1961, par la publication du Jolly qui, poursuivant les fiches interrompues en 1889 par Robert et Cougny, confirme la présentation que le Dictionnaire des parlementaires faisait des députés.

Mais, plus fondamentalement, ce succès tient à l'adéquation entre les intérêts politiques des parlementaires et la gestion symbolique que le «Robert et Cougny " proposa à ce groupe. Comment ne pas souscrire à une entreprise qui éternise la fonction parlementaire, unifie le groupe, objective et universalise son capital spécifique, etc., bref, réifie le collectif que formeraient les parlementaires, cet «en soi» qui incline le mandant à la délégation et l'observateur à l'objectivisme?

L'effet de cette réification est de convier l'analyste à pérenniser l'image du parlementaire en lui assignant le point de vue à partir duquel cette image s'est

74. L'écart entre le doyen (Pyat) et le benjamin (Poincaré) des nouveaux élus de cette législature (dont la moitié avaient moins de 30 ans en 1870) était de 50 ans! Cette différence laisse entendre tout le travail de reconstruction nécessaire à l'analyse prosopographique d'une seule législature afin de tenir compte des différences qui en découlent sur le plan de la formation politique, de la structure des positions sociales d'origine, etc.

75. Il est toujours délicat d'attribuer à ces déclarations une logique plutôt chronologique ou plutôt stratégique. Notons seulement que sur les 58 députés possédant l'attribut «maire», $25 \%$ évoquent cette qualité au premier rang et $60 \%$ aux deux premiers, de même que seulement $27 \%$ des 95 conseillers généraux de l'effectif placent cet attribut au premier rang. 
constituée. La familiarité avec les dictionnaires biographiques, acquise par le politiste ou l'historien au cours de ces multiples exercices où la cohérence entre ce que font les députés et ce qu'ils sont est mise à l'épreuve, place souvent ces derniers dans un rapport de reproduction savante de la population construite. En effet, de telles interrogations, menées à partir des sources et des principes qui construisent la population étudiée, conduisent souvent à mesurer la cohésion du groupe ainsi constitué. Ainsi, c'est paradoxalement à la faveur des démonstrations de légitimité scientifique (la prosopographie, par exemple) que l'analyse savante se place le plus sûrement dans le sillage du point de vue constitutif de la population qu'elle entend étudier.

Hervé Fayat et Nathalie Bayon sont membres du Groupe d'analyse politique de l'Université Paris X-Nanterre 